\title{
Jeitinho as a Coping Strategy Used by Brazilian International Students for Dealing with Acculturative Stress
}

\author{
Silvia Alves Nishioka \\ Defne Akol \\ Teachers College, Columbia University
}

\begin{abstract}
This mixed-method study examined whether Brazilian students in the US use an indigenous problem-solving practice, jeitinho, as a coping strategy for acculturative stress. Forty-two participants answered an online survey, which was a culmination of demographic information, the Acculturative Stress Scale for International Students, BriefCOPE, jeitinho questionnaire, and three open-ended questions about how Americans may perceive jeitinho. Results showed that a negative dimension of jeitinho was associated with nonadaptive coping strategies, but it was not a significant predictor of acculturative stress. Qualitative themes highlighted the multidimensionality of jeitinho, which could help in students' adjustment (positive) or reinforce stereotypes and prejudices (negative) against Brazilians. Findings elucidate the complexity of jeitinho Brazilian students can be mindful about and when to use it to avoid stereotyping.
\end{abstract}

Keywords: acculturative stress, Brazilian, coping, jeitinho

The globalization of the economy and technological development has increased the demand for highly educated professionals who are competent in establishing international partnerships and collaborations (British Council, 2012). As such, there has been an increase in the number of people interested in studying abroad, which both creates unique opportunities to immerse oneself in a different society and culture and also challenges one to quickly adjust to a new environment.

A study focusing on international students' mental health in the United States found a high prevalence of mental health difficulties: $43 \%$ of 551 participants 
reported at least one emotional or stress-related problem that affected their academic performance (Hyun, Quinn, Madon, \& Lustig, 2007). International students may experience stressful situations that require clinical attention, as they have to adjust not only to college but also to a new language and culture (Olivas \& Li, 2006). Thus, clinicians are challenged to incorporate multiple perspectives in their practice to address specific psychological needs of international students, such as acculturative stress, defined as the stress resulting from the process of adapting to a different culture (Berry, Kim, Minde, \& Mok, 1987; Holm-Hadulla, \& Koutsoukou-Argyraki, 2015).

To thrive both academically and socially in the new country, international students may employ a variety of strategies that are culturally driven but are not readily understood or validated by the mainstream culture (e.g., text-borrowing practices; Amsberry, 2009). By identifying and acknowledging such cultural strategies, clinicians may better support students' adjustment. Practices such as the Brazilian jeitinho, defined as a "clever dodge" to solve problems (Da Matta, 2001), could be used by international students in their adjustment process. For instance, Brazilian students may use jeitinho to find alternative methods to finish an important experiment despite not having all the necessary instruments. Yet, there is a dearth in the literature about such indigenous practices and how they are employed.

Little is known about the characteristics of certain subgroups of students or how they adjust to American educational institutions. According to the Open Doors Report (Institute of International Education, 2016), Brazil represented $2.4 \%$ of all international students in 2015, which is the largest student population from a Latin American country in the US. This number has increased due to assistance from Brazilian governmental programs; for instance, more than 5,000 Brazilians received governmental scholarships to study in the US in 2013 (Ministério da Educação, 2013), and it is one of the most promising markets for university recruitment (Fang, Roy, \& Ortiz, 2015). Nonetheless, there is a lack of studies focusing on Brazilian students and their acculturation in the US. This mixed-method study aimed at examining how Brazilian students employ coping strategies while adapting to the US; specifically, how may the indigenous practice, jeitinho, be used as a coping strategy for acculturative stress?

\section{LITERATURE REVIEW}

\section{Acculturation and Acculturative Stress}

Brazilians and other international students often lack much of the cultural background that would help them adjust to American universities. They are rapidly immersed in the educational context while feeling pressured to succeed, which may increase their vulnerability to stress (Holm-Hadulla \& Koutsoukou-Argyraki, 2015). Acculturation is a concept that describes the process of adjustment that international students go through. It can be defined as the dynamic process of cultural and psychological transformation experienced by a person when in contact with a new culture (Berry, 2006; Birman \& Simon, 2013). This concept has been extensively studied among immigrant and refugee populations to clarify the psychological processes related to living in a new country. A meta-analysis evaluating the 
relationship between acculturation and mental health showed that, overall, acculturation to the host culture was positively associated with positive mental health outcomes (Yoon et al., 2013).

Despite being related to positive outcomes, acculturation may be a stressful process. This experience has been conceptualized as acculturative stress, or the strain experienced by a person while acculturating to a new culture (Berry et al., 1987). Studies have shown that the various challenges international students face related to academic performance and social interactions significantly increase their stress (Gebhard, 2012; Yakunina, Weigold, Weigold, Hercegovac, \& Elsayed, 2013). Sandhu and Asrabadi (1994) developed the Acculturative Stress Scale for International Students (ASSIS) to further investigate the concept in this population. The instrument has shown good validity and reliability for international student samples, which improves research with this population (Constantine, Okazaki, \& Utsey, 2004; Yakunina et al., 2013).

Despite their need for mental health support, international students may encounter greater barriers to access treatment, as clinicians may lack certain skills such as providing services in languages other than English (Holm-Hadulla \& Koutsoukou-Argyraki, 2015; Prince, 2015). Considering this, Mori (2000) highlighted that counseling centers should understand the unique characteristics and needs of international students to better serve and support them.

International students experiencing acculturative stress may use more or less adaptive responses to cope. Gebhard (2012) found that students employed behaviors that could be described as facilitators or barriers to their adaptation. Participants expressed that having reminders of their home culture, socializing with other students, and trying to understand and engage with American culture were helpful behaviors. On the other hand, expecting others to adapt to their needs, constantly complaining, and withdrawing from the new culture were considered barriers to adaptation (Gebhard, 2012). Another study observed that certain attitudes toward cultural diversity, such as openness to differences and having contact with diverse people, were associated with significantly lower acculturative stress in a sample of 336 international students in the U.S. (Yakunina et al., 2013). These results emphasize that international students' attitudes and behaviors may promote their acculturation process, thus lowering their acculturative stress.

Coping can be defined as the way one responds to an adverse event and the consequent distress from it (Carver \& Connor-Smith, 2010). Studies have shown the importance of coping to promote physical and psychological health (for a metaanalysis, refer to Penley, Tomaka, \& Wiebe, 2002). There are clinical benefits to fostering coping responses in individuals going through stressful situations. Consequently, it is appropriate to study further the specific responses international students employ to overcome adversity linked to their adjustment to universities.

\section{Brazilian Students and Jeitinho in Brazilian Culture}

A study about mental health experiences of Latinos (mainly from Puerto Rico, Dominican Republic, El Salvador, and Guatemala) and Brazilian immigrants found that the groups differed significantly regarding their help-seeking behaviors for 
mental problems (Sánchez et al., 2014). Among Brazilians, participants who were more acculturated were more likely to endorse treatment-seeking behaviors. This trend was not observed among the other Latino groups, suggesting that people from Latin American countries may approach mental health problems differently. Indeed, Tosta (2004) proposed that Brazilian identity is distinguished from Latino identity. Most Brazilians do not identify themselves as having Hispanic (Spanish) origins, since Brazil was mainly influenced by Portuguese culture and has Portuguese as the official language. The author argued that Brazil has historically assumed an isolated position regarding the other Latin American countries' political and cultural influences. Therefore, Brazilians may have cultural practices and beliefs that are not shared with other Latino cultures. These cultural practices and beliefs are salient to Brazilian populations in how they relate to other people and handle daily problems. For these reasons, the Brazilian population may be better examined as a separated group.

An example of a salient practice in the Brazilian culture that is less relevant to other Latino groups is jeitinho. Sociologists and anthropologists have described jeitinho (pronounced jaytcheenyoo) brasileiro as a "clever dodge," a way to "get things done" by bending or breaking norms mainly based on personal relations (Da Matta, 2001; Duarte, 2006). Jeitinho, as a problem-solving strategy, is considered an indigenous construct very common in Brazil that intrigues researchers due to its ambiguity. The origins of jeitinho are unclear, but it was linked to overbureaucratized, rigid, and excessively formal contexts that characterize many Brazilian institutions, especially public institutions (Duarte, 2006). Jeitinho would be a way to deal with these contexts and, most importantly, is considered part of the Brazilian identity (Barbosa, 1992; Da Matta, 2001). It is a widespread practice present in a variety of social contexts and easily recognized by the expression: "Dá um jeitinho para mim" ([Give me a jeitinho]; Duarte, 2006).

Ferreira, Fischer, Porto, Pilati, and Milfont (2012) tried to explore the concept of jeitinho, capturing its intrinsic ambiguity. The authors developed and tested scenarios depicting the use of jeitinho (see Appendix). They distinguished three underlying dimensions: corruption (i.e., morally questionable ways to solve a problem), creativity (i.e., new and creative ways to solve a problem), and social-norm breaking (i.e., bypassing social norms to solve a problem). The results underscore the complexity of Brazilian culture and its practices; participants acknowledged that jeitinho might produce negative consequences and be negatively perceived by other people, and, at the same time, they recognized that most Brazilians engage in it (Ferreira et al., 2012).

Research found jeitinho to be comparable to other indigenous constructs: guanxi in China, wasta in Lebanon, and "pulling strings" in the United Kingdom (Smith, Huang, Harb, \& Torres, 2010). Each construct was considered to have a culturalspecific practical value to solve problems. Considering the perception that jeitinho is part of Brazilian identity, it is relevant to investigate its use and implications in contexts that may not recognize it as a valid strategy. In organizational settings, research has examined the influence of jeitinho in business in Brazil as negative moral implications (Dennis \& Stroh, 1997; O'Keefe \& O'Keefe, 2004). Similar to other cultural practices such as guanxi in China, jeitinho is heavily based on informal 
connections and social influence, which can be seen as a negative characteristic by cultures that value formal structures and organizations (Smith et al., 2010). It is possible that non-Brazilians perceive jeitinho negatively and disapprove its use in academia and workplaces, but little is known about these perceptions.

Research involving Brazilian immigrants highlights the challenges they face that have negative repercussions to mental health. Studies observed that Brazilian immigrants may be discriminated against, which intensifies the stress during adaptation (Oliveira et al., 2016). To cope with such adverse situations, Brazilians may employ a variety of strategies including jeitinho. Hafemeister (2005) interviewed Brazilian immigrants in Miami and found that they used jeitinho as a critical strategy when confronted with difficulties related to their acculturation to the US. Many participants reported being able to thrive due to their ability of dar um jeitinho ([give a jeitinho]; Hafemeister, 2005). Jeitinho has an evident value as a strategy that is often employed by Brazilians, yet little is known about its use outside Brazil. Although international students do not face the same challenges as immigrants do, the former still have to quickly adapt to a new culture when being pressured to perform academically, which may result in increased stress requiring the use of strategies such as jeitinho.

To date, few studies have focused on the Brazilian immigrant population (Oliveira et al., 2016), and none have focused on Brazilian international students investigating their difficulties in the host country and the strategies, including jeitinho, that may help them in their adaptation process. To address this gap in the literature, this study aimed to answer the following research questions: (a) What are the coping strategies used by Brazilian international students in the United States for acculturative stress? (b) Is jeitinho used as a coping strategy to reduce acculturative stress? And, (c) how do non-Brazilians perceive the use of jeitinho by Brazilian students? It was hypothesized that: (a) jeitinho would be a significant coping strategy among Brazilian students for acculturative stress; (b) lower levels of acculturative stress would be associated with adaptive coping strategies and jeitinho; and (c) American professors and colleagues would perceive jeitinho as a negative practice.

\section{METHOD}

\section{Study Design}

This study used a mixed-method design to address the research questions. The qualitative measures aimed to increase knowledge regarding the Brazilian international student population and their levels of acculturative stress, coping strategies, and use of jeitinho to compare between participants. Due to the lack of objective measures and the exploratory nature of the investigation, the authors formulated three open-ended questions to examine the perceptions of non-Brazilians about Brazilians using jeitinho based on previous studies (Dennis \& Stroh, 1997; O'Keefe \& O'Keefe, 2004). Additionally, the qualitative material could further elucidate the quantitative results. 


\section{Participants}

The present study considered an international student any individual who is a non-U.S. citizen or permanent resident who came to the US primarily to study. Participants were recruited through a Brazilian student association. This association focuses on disseminating relevant information for undergraduate and graduate Brazilian students studying in the US through their website and social media. Brazilian students can subscribe to their website and receive this information or access it directly on their social media pages. The association announced the study to potential participants on their email newsletter and social media pages. Participation was voluntary and involved responding to an anonymous survey. A gift card raffle was conducted for those who completed all the questions. Inclusion criteria were selfidentify as Brazilian, be a current student in a college or university in the United States, and voluntarily accept to participate in the study. Forty-two $(n=42)$ Brazilian students in the U.S. completed the quantitative scales, and from those, twenty-eight $(n=28)$ also answered the qualitative questions included in the analyses. The Teachers College Institutional Review Board approved this study prior to data collection. Data was collected from November 2016 to January 2017.

\section{Measures}

Participants completed demographic information, the ASSIS, and the BriefCope inventory. Additionally, the jeitinho questionnaire (Ferreira et al., 2012) and three open-ended questions developed by the authors were used to examine jeitinho.

The ASSIS (Sandhu \& Asrabadi, 1994) has 36 items rated using a 5-point Likerttype scale, describing different possible stressful situations such as, "Multiple pressures are placed upon me after migration." Seven factors are considered: perceived discrimination (eight items), homesickness (four items), perceived hate (five items), fear (four items), stress due to change/culture shock (three items), guilt (two items), and miscellaneous (10 items). A total score higher than 109 is considered in need of clinical attention. In this study, the instrument reliability was $\alpha=.93$.

The BriefCope (Carver, 1997) assesses both adaptive and nonadaptive coping strategies. Maroco, Campos, Bonafé, Vinagre, and Pais-Ribeiro (2014) developed a Brazilian version of the BriefCOPE translating and validating the items to this population. The instrument has 28 items (e.g., "I concentrate my efforts in doing something about it," "I give up the attempt to get what I want") describing different coping strategies rated according to a 5-point Likert-type scale. Scores for adaptive and nonadaptive strategies are calculated separately; higher scores indicate a higher frequency of that type of strategy. In this study, the instrument reliability was $\alpha=.83$.

Participants responded to a questionnaire evaluating jeitinho among Brazilians composed of 19 hypothetical scenarios of the use of jeitinho (Ferreira et al., 2012). Participants rated how likely they would behave in the described way (i.e., using jeitinho $)$. Answers range from 0 to $10(0=$ very unlikely and $10=$ very likely $)$. Three jeitinho dimensions were represented: corruption, creativity, and norm breaking. According to the developers, corruption refers to a problem-solving strategy that involves illicit methods. Creativity refers to an innovative or creative way of solving 
a problem without violating social or legal norms. Norm-breaking refers to strategies that bypass some social norms to solve a problem. Examples of these scenarios are found in the Appendix. Scores are calculated by summing the responses; higher scores indicate higher likelihood of using jeitinho as a problem-solving strategy. For corruption dimension scores range from 0 to 60 , for creativity scores range from 0 to 70 , and for norm-breaking from 0 to 60 . In this study, the instrument reliability was $\alpha=.76$.

Finally, participants answered three open-ended questions developed by the authors on the online survey to gather qualitative data on how Americans may perceive jeitinho: (a) "If your professor at your college/university knew you were using jeitinho to solve a problem, in your opinion, what would s/he think? Would this influence their impressions about you?"; (b) "If a classmate at your college/university knew you were using jeitinho to solve a problem, in your opinion, what would s/he think? Would this influence their impressions about you?"; and (c) "If an American friend/colleague knew you were using jeitinho to solve a problem, in your opinion, what would s/he think? Would this influence their impressions about you?"

\section{Data Analyses}

Following a mixed-method design, first, quantitative data and qualitative data were analyzed separately. Then, the findings were integrated yielding a more holistic understanding of the use of jeitinho as a coping strategy for acculturative stress in Brazilian international students.

Quantitative data (i.e., ASSIS, BriefCOPE, and jeitinho questionnaire) was analyzed through SPSS using the total scores of each participant in each measure. First, participants $(n=42)$ were divided into two groups according to their ASSIS total score: Scores below or equal to 109 were considered low acculturative stress (LAS; $n=34$ ) and scores higher than 109 were considered high acculturative stress (HAS; $n=8$ ). Scores from BriefCOPE were divided into adaptive coping (AC) and nonadaptive coping (NC). Results of jeitinho questionnaire were analyzed in its three different dimensions: corruption (CP), creativity (CR), and norm-breaking (NB). Higher scores indicated higher likelihood the participant used jeitinho in the described way. We conducted an analysis of variance (ANOVA) to compare the measures' scores between LAS and HAS groups. Multiple regression analyses were conducted to determine significant associations between LAS and AC, NC, CP, CR, and NB while controlling for the participant's age, gender, and level of education.

Qualitative data (i.e., answers to three open-ended questions about jeitinho from the online survey) were categorized using thematic analysis (Braun \& Clarke, 2006) to capture the common themes across participants. Following a first reading of all responses, initial themes were generated for each participant (total of 28 participants, 84 responses). Then, common themes across responses were searched and elaborated to accurately capture distinct perspectives related to the research question. The themes were reviewed and defined using significant instances from the dataset. Quantitative and qualitative analyses were then integrated to provide a more holistic comprehension of the present research theme. 


\section{RESULTS}

The present study aimed to: (a) identify the coping strategies used by Brazilian international students in the U.S. for acculturative stress; (b) assess whether jeitinho is used as a coping strategy to reduce acculturative stress; and (c) how non-Brazilians perceive the use of jeitinho by Brazilian students. It was expected that: (a) jeitinho would be a significant coping strategy among Brazilian students for acculturative stress; (b) lower levels of acculturative stress would be associated with adaptive coping strategies and jeitinho; and (c) American professors and colleagues would perceive jeitinho as a negative practice.

\section{Quantitative Analysis}

Table 1 summarizes the participants' demographic characteristics divided by high acculturative stress (HAS) and low acculturative stress (LAS) groups. Overall, participants were aged between 27 and 31 years, identified as female, and were single. They were all Brazilian nationals and asked to identify their ethnic background according to Brazilian census categories (e.g., non-Hispanic European, multiracial, Brazilian native).

Table 1: Demographic Information by Level of Acculturative Stress

\begin{tabular}{lcccc}
\hline & \multicolumn{2}{c}{ HAS } & \multicolumn{2}{c}{ LAS } \\
\cline { 2 - 5 } Variables & $n=8$ & $\%$ & & \\
\hline Age & 0 & 0 & 6 & 17.6 \\
18 to 22 years & 2 & 25 & 6 & 17.6 \\
23 to 27 years & 4 & 50 & 18 & 52.9 \\
27 to 31 years & 2 & 25 & 1 & 2.9 \\
32 to 36 years & 0 & 0 & 2 & 5.9 \\
37+ years & & & & \\
Gender & 2 & 25 & 10 & 29.4 \\
Male & 6 & 75 & 24 & 70.6 \\
Female & & & & \\
Ethnicity & 1 & 12.5 & 0 & 0 \\
African & 1 & 12.5 & 2 & 5.9 \\
Asian & 3 & 37.5 & 17 & 50 \\
Brazilian Native & 2 & 25 & 6 & 17.6 \\
European Hispanic & 5 & 62.5 & 9 & 26.5 \\
$\quad$ European non-Hispanic & 5 & 62.5 & 6 & 17.6 \\
$\quad$ Multiracial & & & & \\
Marital Status & 3 & 37.5 & 22 & 64.7 \\
$\quad$ Single & 3 & 37.5 & 10 & 29.4 \\
$\quad$ Married & 2 & 25 & 1 & 2.9 \\
Living together & & &
\end{tabular}




\begin{tabular}{lcccc}
\hline & \multicolumn{2}{c}{ HAS } & \multicolumn{2}{c}{ LAS } \\
\cline { 2 - 5 } Variables & $n=8$ & $\%$ & $n=34$ & $\%$ \\
\hline Divorced & 0 & 0 & 1 & 2.9 \\
Current level of education & & & & \\
$\quad$ College student & 0 & 0 & 9 & 26.5 \\
Master's student & 0 & 0 & 5 & 14.7 \\
MBA student & 2 & 25 & 7 & 20.6 \\
Doctoral student & 6 & $75^{*}$ & 12 & 35.3 \\
Post-doctorate student & 0 & 0 & 1 & 2.9 \\
Residence in the U.S. & & & & \\
West Coast & 3 & 37 & 3 & 9 \\
East Coast & 3 & 38 & 15 & 44 \\
Central & 1 & 13 & 13 & 38 \\
South & 1 & 12 & 3 & 9 \\
\hline
\end{tabular}

Note. HAS $=$ high acculturative stress; LAS $=$ low acculturative stress. ${ }^{a}$ More than one option was possible; ${ }^{*} p<.05$

Table 2 shows the students' scores in each measure and significant differences between the groups with high and low levels of acculturative stress. Most participants reported low levels of acculturative stress $(81 \%)$; only a small number of participants reported being highly distressed (19\%). Most acculturative stress factors were significantly different between the two groups with the exception of homesickness. Level of education emerged as the only significant difference in demographics; doctoral students represented $75 \%$ of those reporting HAS compared with $12 \%$ of those with LAS. No other significant differences were noted regarding the demographics.

Table 2: Analyses of Variance Comparing High and Low Levels of Acculturative Stress

\begin{tabular}{lcccc}
\hline & \multicolumn{2}{c}{ HAS } & \multicolumn{2}{c}{ LAS } \\
\cline { 2 - 5 } Measure & $M$ & $S D$ & 81.3 & 17.71 \\
\hline ASSIS (total) & 117.6 & 7.17 & 17.2 & \\
Discrimination & $26.5^{* *}$ & & 12.2 & \\
Homesickness & 14.9 & & 8.6 & \\
Perceived hate & $13.8^{* *}$ & & 6.6 & \\
Fear & $10.9^{* *}$ & & 6.8 & \\
Cultural shock & $10.5^{* *}$ & & 4.5 & \\
Guilt & $6.4^{*}$ & & 24.6 & \\
Miscellaneous & $34.6^{* *}$ & & 58.9 & 8.75 \\
Adaptive coping & 56.1 & 7.47 & $23.3^{*}$ & 3.94 \\
Non-adaptive coping & $32.6^{*}$ & 5.12 & $0.27 *$ & 0.41 \\
Jeitinho corruption & $0.62^{*}$ & 0.39 & 4.41 & 0.99 \\
Jeitinho creativity & 5.05 & 1.10 & 1.74 & 1.20 \\
Jeitinho norm-breaking & 1.98 & 0.93 & & \\
\hline
\end{tabular}


Note. HAS = high acculturative stress; LAS = low acculturative stress; ASSIS = Acculturative Stress Scale for International Students. $* p<.05 ; * * p<.01$

Regarding coping strategies, two significant differences were observed between participants with HAS versus LAS: Non-adaptive coping and jeitinho corruption scores were higher for those with HAS. Despite Hypothesis 2, no differences were observed in the report of adaptive coping, jeitinho creativity, and jeitinho normbreaking. Although participants with HAS employed adaptive strategies, they tended to use significantly more nonadaptive coping compared with participants with LAS. Similarly, the corruption dimension of jeitinho was more often employed than creativity and norm-breaking by participants with HAS. Indeed, correlation analyses (not reported here due to space limitations) showed that high scores of acculturative stress were significantly correlated with nonadaptive coping and corruption dimension $(r=.37, p<.05)$. Although not significantly different, the means of the three components of jeitinho were higher among participants with higher levels of stress in comparison to those with lower levels. This small difference may indicate a tendency that participants who employed more jeitinho were also more distressed.

Based on multiple regression analyses (Table 3), primary factors associated with LAS were higher utilization of adaptive coping strategies and lower utilization of nonadaptive coping strategies. The influence of coping strategies on acculturative stress was independent of demographic factors, including level of education. Although doctoral students scored higher on acculturative stress measures than the rest of the students, the only significant predictor of acculturative stress was the use of coping strategies for all students. It is possible that doctoral students have a different experience from undergrads and masters-level students increasing their levels of acculturative stress. Perhaps the multiple roles doctoral students have to play (student, researcher, lecturer) may pressure them more compared with other students.

Table 3: Coping Strategies and Jeitinho as Predictors of Low Levels of Acculturative Stress

\begin{tabular}{lccc}
\hline & $B$ & $S E B$ & $\beta$ \\
\cline { 2 - 4 } Age & -.022 & .054 & -.054 \\
Gender & .123 & .109 & .138 \\
Level of education & -.078 & .045 & -.236 \\
Adaptive coping & .016 & .006 & $.341^{*}$ \\
Non-adaptive coping & -.046 & .010 & $-.641^{* *}$ \\
Jeitinho corruption & -.095 & .141 & -.101 \\
Jeitinho creativity & -.034 & .050 & -.089 \\
Jeitinho norm-breaking & -.007 & .055 & -.021 \\
\hline
\end{tabular}

Note. $R^{2}=3.90 ; * p<.05 ; * * p<.01$

The findings show that participants from both groups use adaptive and nonadaptive coping strategies. However, participants with HAS tend to employ nonadaptive coping strategies more frequently than those with LAS, which may exacerbate their stress. The dimensions of jeitinho were not significantly associated 
with acculturative stress in this sample. The data does not support the initial hypothesis that jeitinho would be a relevant coping strategy for acculturative stress among Brazilian students.

\section{Qualitative Analysis}

Thematic analysis was used to examine the qualitative data that was gathered through the open-ended questions. We aimed to explore participants' impressions of how American professors, classmates, and friends might perceive jeitinho. Eightyfour $(N=84)$ responses from 28 participants yielded three main themes, namely: "impressions of jeitinho," "negative aspects of jeitinho," and "positive aspects of jeitinho" (Figure 1).

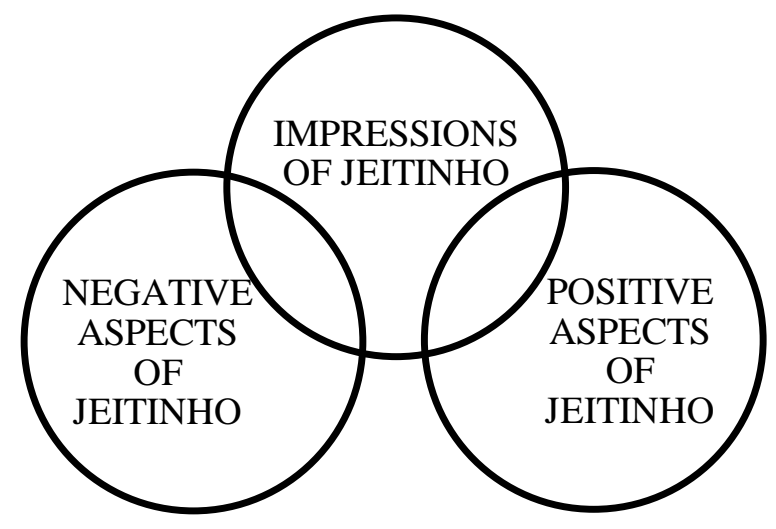

\section{Figure 1: Themes Found in the Qualitative Analysis}

The theme, "impressions of jeitinho," included most responses (49\%) and emphasized the complexity of the strategy. Participants mentioned that there are different uses, interpretations, and consequences associated to jeitinho. It can be perceived as negative or positive; "it depends" how it is used. Jeitinho can be "cheating," doing something clearly "illegal" that would result in severe consequences for the person. Alternatively, jeitinho can be a "legitimate way" to solve problems leading to positive consequences. For instance:

If I am being creative, accommodating problems, and trying to create a better working environment, I think jeitinho is seen as a great thing. On the other hand, if I am using jeitinho to fool members of the university, I think they would not accept this kind of behavior and see me in a bad light.

Responses also stated that if Brazilians employed jeitinho in a positive way, Americans would perceive it positively. However, if it is bending norms or illicit, Americans would perceive it negatively and there will be implications to it. Therefore, participants pointed that they are mindful about when to use jeitinho to 
avoid this negative connotation. For example, "I try to use the 'jeitinho' wisely, so that others are not impacted in a negative way. If I feel I might be taking advantage of anyone, I will refuse to do it." Therefore, Brazilian students are aware of the multiple dimensions of jeitinho and did not use it instinctively in the United States as they may do in Brazil where it is widely accepted.

The second theme was "negative aspects of jeitinho" including responses (43\%) that mentioned negative perceptions Americans have about jeitinho. Supporting the study hypothesis, Brazilian students mentioned they would be considered "untrustworthy," "lazy," "cutting corners," and "looking for the shortest path" if they used jeitinho. For instance:

In some ways, the Brazilian jeitinho is dishonest and simply wrong. It does not go in accordance with American values. It would be hard for them to accept it because they do not share of the same cultural upbringing and whenever you try to explain it, it sounds like you are just looking for the shorter path, which can give you an image of lazy.

Some responses highlighted that jeitinho could be an illicit strategy, which is associated to the corruption dimension of the quantitative questionnaire. Moreover, there would be severe consequences, "disciplinary actions," affecting the student's academic performance and relationship with non-Brazilian professors, classmates, and friends. These impressions negatively impact relationships and potentially harm the impressions Americans have of Brazil in general. Indeed, 13\% of all responses stated that negative impressions linked to jeitinho could be generalized to all Brazilians as part of the culture. This would confirm stereotypes and prejudices against Brazilians. For instance:

It would definitely influence [the professor's] impressions about me and Brazilian people in general. The "jeitinho" is usually some "illegal" trick or shortcut to do something, and it can give the impression of laziness.

These statements illustrate the concerns Brazilian students may have in using jeitinho in their academic settings, as they fear to be discriminated against or to reinforce a negative stereotype of Brazil. This specific topic may have important implications for the students' adaptation to American universities.

Participants also reported that Americans might perceive jeitinho positively. These responses (7\%) were included in the theme "positive aspects of jeitinho." In a more nuanced way, participants explained that Americans would praise the creativity in jeitinho as "thinking outside of the box" and showing "resourcefulness." For example, "jeitinho doesn't have to be illicit or dishonest, there are plenty of ways that a problem can be solved creatively within the confinements of morality and legality." Moreover, participants emphasized how jeitinho could be helpful in solving problems. They mentioned that their colleagues often admired them or were inspired by their ability to overcome difficulties. These positive consequences were observed in instances like: "My friends are often impressed on how I can solve some problems using jeitinho. They believe that is extremely creative and lots of them wished to know how to get away using it. I was already praised for that." 
The findings partially support the initial hypothesis that Americans would perceive jeitinho negatively. The theme "negative aspects of jeitinho" supports this hypothesis. However, the themes "impressions of jeitinho" and "positive aspects of jeitinho" point to the complexity of the construct and how it is linked to the situation that is employed. Regarding this, American professors and students may be very strict in condemning such practice if it is used against the rules or to take advantage of others. At the same time, they may perceive it as a good skill and even desire they were able to use jeitinho in a creative way. Overall, perceptions about jeitinho may include discrimination and prejudice against Brazilians and Brazilian culture, but also admiration and recognition of the usefulness of the strategy. Noteworthy, the complexity of jeitinho could not be captured only by the quantitative measure, especially concerning the different implications of using it.

\section{DISCUSSION}

The present study was one the first investigations looking into levels of acculturative stress among Brazilian international student in the US and the coping strategies they use, including the indigenous practice, jeitinho. No significant relationship was found between the dimensions of jeitinho and levels of acculturative stress. The findings did not support the initial hypothesis that jeitinho would be a relevant coping strategy for acculturative stress. One explanation for these results is that jeitinho is a core aspect of Brazilian identity (Da Matta, 2001). Duarte (2006) argued that over-bureaucratic institutions in Brazil might have accentuated the practical relevance of this strategy that helps people to find "unofficial" solutions to their problems bypassing bureaucracy and rules. Supporting this idea, qualitative responses in this study pointed to the close relationship between jeitinho and what is seen as being "Brazilian." Specifically, participants mentioned stereotypes that Americans may hold regarding the Brazilian identity. Indeed, jeitinho is heavily based on social influence and can be seen as a negative characteristic by cultures that value the respect for formal structures and organizations (Smith et al., 2010). Consequently, Brazilian students may decide not to use jeitinho in the U.S. to avoid negative perceptions.

Due to its possible association to Brazilian identity, jeitinho may be more linked to enculturation than acculturation or acculturative stress, which would explain its nonsignificant relationship with acculturative stress. Research has shown that acculturation and enculturation are related but pertain to different dimensions and are independently related to mental health outcomes (Yoon et al., 2003). Also, studies have found that enculturation was positively related to good mental health in African American and Latino individuals (Yoon et al., 2003). As emphasized by the study's qualitative findings, jeitinho is a complex construct and involves both positive and negative connotations depending on how it is employed, with the uncertainty of whether it will have a positive effect on mental health or not. Future investigations could aim to evaluate the possible relationship between enculturation and jeitinho.

Most participants reported low levels of acculturative stress. Those who were more stressed also employed nonadaptive strategies more frequently than those less stressed. Similarly, participants with higher levels of stress were more likely to endorse jeitinho corruption, suggesting that the corruption dimension of jeitinho 
could be another nonadaptive coping behavior particularly used by Brazilian international students. As some participants mentioned in their qualitative responses, they are mindful of the use of jeitinho in the US, avoiding its negative consequences. Perhaps Brazilians who are not as aware of such consequences may use jeitinho instinctively and experience negative perceptions by Americans which, in turn, would increase their acculturative stress.

The use of nonadaptive behaviors was also found in previous research with international students (Gebhard, 2012) in which participants recognized that they would behave in ways that were not helpful to their adjustment. In the present study, participants described negative aspects and consequences of using jeitinho. Many of the qualitative answers pointed to the "illegal" and "wrong" side of using jeitinho and how it would negatively impact their interpersonal relationships. Some descriptions included in the theme "negative aspects of jeitinho" were aligned with the conceptualization of the corruption dimension from the quantitative questionnaire. Furthermore, qualitative responses indicated that Americans reacted to jeitinho corruption negatively, possibly because it goes against their cultural values. Participants recognizing such cultural variance and negative perceptions in their academic settings may consider inappropriate to use jeitinho. Hence, it is possible that students with HAS in employing more adaptive behaviors that would promote their adjustment. Perhaps, students who are more stressed may use jeitinho as they do in Brazil, where the practice is widely accepted. In the US, the ambiguity of jeitinho may not be understood or accepted in the same way (Ferreira et al., 2012), leading to more stressful situations for Brazilian students who use it compared to when they are in Brazil.

Participants mentioned being aware of the cultural differences between American and Brazilian values and consciously deciding about the appropriate situations to use jeitinho to prevent or decrease discrimination. However, as highlighted by other studies, the anticipation of discrimination due to cultural differences may have adverse effects on the individual's mental health by itself(Yoon et al., 2003). Therefore, the perception that jeitinho would have a negative impact on them and other Brazilians could be in itself a barrier to adaptation and affect mental health outcomes. This could reinforce the use of indigenous practices that come "instinctively" to the international student increasing stress and hindering adjustment. Most participants reported low levels of acculturative stress, yet this could be an underlying issue for those who had higher scores that would need clinical attention. Further investigation is needed to better understand the effects of nonadaptive coping strategies on acculturative stress and overall adjustment; particularly, how indigenous practices may be employed as nonadaptive strategies.

Some qualitative responses emphasized the positive aspects of jeitinho that is admired by non-Brazilians. These statements described that professors and colleagues are impressed by the Brazilian ability to overcome problems and could be linked to jeitinho creativity of the questionnaire. Moreover, participants expressed that the situation is relevant to determine how jeitinho is perceived. These findings did not support the initial hypothesis that non-Brazilians would perceive jeitinho only negatively. 
Finally, we found that doctoral students reported significantly higher levels of acculturative stress. It is possible that the academic challenges linked to doctoral studies increase the stress experienced by international students who have to handle with multiple roles student, researcher, and often lecturer or teacher assistant. Yet, educational level was not a significant predictor of acculturative stress. Future investigations could focus on this specific population analyzing possible factors associated with higher levels of acculturative stress and potential interventions to support these students.

Some limitations should be considered when interpreting the results. First, this was an online survey conducted with a small sample of students; future studies should involve a larger sample and also administer in-person interviews gathering more details about the use of jeitinho in American academic settings. In-depth interviews could further explore prejudices and stereotypes that exist towards this practice. Secondly, this study involved only Brazilian participants' impressions of how Americans would perceive jeitinho. It would be important to include non-Brazilian participants to express their perceptions about jeitinho. Finally, the only measure of acculturative stress was the ASSIS, and other measures of acculturation and enculturation could have been included and tested regarding its relationship with jeitinho.

\section{Conclusion and Implications}

This study opens a new set of questions about the use of culture-specific practices by international students. The advance of such area would provide important information to better support and foster healthy adaptation of this population to their host country.

The present study expanded the knowledge on the levels of acculturative stress, coping strategy, and jeitinho. Moreover, it highlighted the complexity of this cultural practice regarding the different perceptions Brazilians and non-Brazilians may hold about it. Overall, Brazilian students reported low levels of acculturative stress, employing adaptive coping strategies to adjust to the US. Additionally, participants reported using jeitinho in its creative dimension, which may help them to solve a diversity of problems increasing their resources to succeed in American universities, such as running experiments despite the lack of adequate equipment. Although jeitinho was not significantly associated with lower levels of stress, it may be a helpful strategy for Brazilian students in their daily lives.

The findings corroborated previous studies (Duarte, 2006; Ferreira et al., 2012) about jeitinho demonstrating the multiple aspects of this practice and its uniqueness to Brazilian culture. Particularly, the findings emphasized that jeitinho could yield different outcomes depending on how it is used. Jeitinho may be linked to Brazilian identity in general and related to enculturation rather than acculturation. Qualitative data added important nuances to the investigation of the use of jeitinho outside Brazil, suggesting that Brazilian students are aware of the different impressions and implications of the use of jeitinho. Such concern may not be present when they are in Brazil because jeitinho is a common and acceptable practice there. Nevertheless, when living abroad, they have to be more mindful about the employment of the 
strategy, avoiding negative perceptions and consequences. Such implications were not observed in previous investigations (Hafemeister, 2005), in which only the positive aspects of jeitinho were discussed.

Another important implication was the possible discrimination against Brazilian students due to jeitinho. Many qualitative responses evoked the concern of being devaluated according to cultural prejudices. Participants were worried that all Brazilians would be perceived negatively because of their use of jeitinho. This concern had twofold consequences: First, at the individual level, the student's interpersonal relationships with professors and colleagues would be affected, and this could impact their academic development. Second, at the community level, the negative impression would be generalized to other Brazilian students, reinforcing a negative stereotype about the group. This second consequence indicates that Brazilian students might fear a more systematic discrimination against Brazilians in American academic settings due to the use of indigenous practices. The relationship between jeitinho and discrimination was not mentioned in previous investigations with Brazilians living outside Brazil (Hafemeister, 2005; Oliveira et al., 2016) and should be further researched. Yet, most participants in this study mentioned the positive aspects of jeitinho, especially in its creative dimension. Possibly, jeitinho as a strategy that creatively finds new ways for solving a problem could be taught and learned as some participants mentioned in their responses.

The data highlights the importance of supporting Brazilian students in their adaptation to the US. Although a small proportion of participants reported being more stressed, those who did also reported a more frequent use of nonadaptive coping strategies and jeitinho corruption. Such strategies may have a detrimental effect on how they are perceived, affecting their adjustment. Mental health services in universities should be culturally competent, factoring in cultural-specific issues and practices such as jeitinho to assist international students in developing more adaptive coping strategies and decreasing stress levels. For instance, counselors could explore and identify how cultural practices may influence their adaptation. Furthermore, student associations and mentorship programs could be a valuable source of support to international students where they could share their difficulties and learn adaptive strategies. For example, in our study, we observed the relevance of understanding in which situations to employ jeitinho; senior students could mentor incoming students helping them to use jeitinho "wisely" given the university context and American culture. Future research should address the limitations of this study such as including more participants, Brazilians and non-Brazilians, conducting in-depth interviews, and assessing enculturation.

The present study shed light on the use of an indigenous practice in Brazil that has not been examined in the context of a different culture that may not accept it as a valid strategy for solving problems. Jeitinho was not found to be significantly associated with acculturative stress because it may be related to enculturation rather than acculturation. The data showed the complexity of this practice and how Brazilians were mindful about using it in appropriate ways and situations to avoid negative impressions. These impressions included discrimination and stereotypes, but positive perceptions and admiration were also mentioned as possible outcomes. These different outcomes depended on the ability of Brazilians to use jeitinho carefully. 
Such ability may require knowledge of American culture and skills to navigate encounters with American professors and colleagues. Therefore, it is important that mental health services and international offices are culturally competent supporting Brazilian students in their specific needs and promoting a better adaptation to the university.

\section{REFERENCES}

Amsberry, D. (2009). Deconstructing plagiarism: International students and textual borrowing practices. The Reference Librarian, 51(1), 31-44.

Barbosa, L. (1992). O jeitinho brasileiro. Rio de Janeiro: Campus.

Berry, J. W. (2006). Acculturation: A conceptual overview. In Bornstein, M. H. \& Cote L. R. (Eds.), Acculturation and parent-child relationships: Measurement and development (pp. 13-30). New York: Routledge.

Berry, J. W., Kim, U., Minde, T., \& Mok, D. (1987). Comparative studies of acculturative stress. International Migration Review, 21(3), 491-511.

Birman, D., \& Simon, C. D. (2013). Acculturation research: Challenges, complexities, and possibilities. In APA handbook of multicultural psychology. Washington, DC: American Psychological Association.

Braun, V., \& Clarke, V. (2006). Using thematic analysis in psychology. Qualitative Research in Psychology, 3(2), 77-101.

British Council. (2012). The shape of things to come: Higher education global trends and emerging opportunities to 2020: Global edition. Retrieved from https://www.britishcouncil.org/sites/default/files/the_shape_of_things_to_come _-_higher_education_global_trends_and_emerging_opportunities_to_2020.pdf

Carver, C. S. (1997). You want to measure coping but your protocol's too long: Consider the brief cope. International Journal of Behavioral Medicine, 4(1), 92100.

Carver, C. S., \& Connor-Smith, J. (2010). Personality and coping. Annual Review of Psychology, 61, 679-704.

Constantine, M. G., Okazaki, S., \& Utsey, S. O. (2004). Self-concealment, social selfefficacy, acculturative stress, and depression in African, Asian, and Latin American international college students. American Journal of Orthopsychiatry, 74, 230-241.

Da Matta, R. (2001). O que faz o brasil, Brasil? (Vol. 7). Rio de Janeiro: Rocco.

Dennis, L. E., \& Stroh, L. K. (1997). A little jeitinho in Brazil: A case study on international management. Journal of Management Education, 21(2), 255.

Duarte, F. (2006). Exploring the interpersonal transaction of the Brazilian jeitinho in bureaucratic contexts. Organization, 13(4), 509-527.

Fang, Y., Roy, M., \& Ortiz, A. A. (2015, May). Top emerging markets for international student recruitment. Retrieved from http://wenr.wes.org/2015/05/top-emerging-markets-international-studentrecruitment.

Ferreira, M. C., Fischer, R., Porto, J. B., Pilati, R., \& Milfont, T. L. (2012). Unraveling the Mystery of Brazilian Jeitinho a Cultural Exploration of Social Norms. Personality and Social Psychology Bulletin, 38(3), 331-344. 
Gebhard, J. G. (2012). International students' adjustment problems and behaviors. Journal of International Students, 2(2), 184-193.

Hafemeister, B. E. (2005). Identity and adaptation of Brazilian immigrants in Miami (Unpublished Master's thesis). University of Miami: Coral Gables, FL.

Holm-Hadulla, R. M., \& Koutsoukou-Argyraki, A. (2015). Mental health of students in a globalized world: Prevalence of complaints and disorders, methods and effectivity of counseling, structure of mental health services for students. Mental Health \& Prevention, 3(1), 1-4.

Hyun, J., Quinn, B., Madon, T., \& Lustig, S. (2007). Mental health need, awareness, and use of counseling services among international graduate students. Journal of American College Health, 56(2), 109-118.

Institute of International Education. (2016, September). Open Doors report. Retrieved from http://www.iie.org/Services/Project-Atlas/UnitedStates/International-Students-In-US\#.V 5FxOgrI2w

Maroco, J., Campos, J. B., Bonafé, F. S., Vinagre, M. D. G., \& Pais-Ribeiro, J. (2014). Adaptação transcultural Brasil-Portugal da escala Brief Cope para estudantes do ensino superior [Brazil-Portugal transcultural adaptation of BriefCope for students in higher education]. Psicologia, Saúde \& Doenças, 15(2), 300-313.

Ministério da Educação. (2013, February). Bolsas concedidas pela CAPES e CNPq, por modalidade e por país, no Programa Ciências sem Fronteiras. Retrieved from http://www.cienciasemfronteiras.gov.br/web/csf/estatisticas-e-indicadores

Mori, S. (2000). Addressing the mental health concerns of international students. Journal of Counseling and Development, 78, 137-144.

O'Keefe, H., \& O'Keefe, W. M. (2004). Business behaviors in Brazil and the USA: Understanding the gaps. International Journal of Social Economics, 31(5/6), 614-622.

Olivas, M., \& Li, C. (2006). Understanding stressors of international students in higher education: What college counselors and personnel need to know. Journal of Instructional Psychology, 33(3), 217.

Oliveira, E. N., Monteiro Neto, F. F., Freire, A. S., Felix, T. A., Moreira, R. M. M., \& Lima, G. F. (2016). Saúde de imigrantes: Estudos com brasileiros baseado em evidências [Immigrant health: Evidence-based studies with Brazilians]. SANARE, 15(1), 74-81.

Penley, J. A., Tomaka, J., \& Wiebe, J. S. (2002). The association of coping to physical and psychological health outcomes: A meta-analytic review. Journal of Behavioral Medicine, 25(6), 551-603.

Prince, J. P. (2015). University student counseling and mental health in the United States: Trends and challenges. Mental Health \& Prevention, 3(1), 5-10.

Sánchez, M., Cardemil, E., Adams, S. T., Calista, J. L., Connell, J., DePalo, A., . . . Rivera, I. (2014). Brave new world: Mental health experiences of Puerto Ricans, immigrant Latinos, and Brazilians in Massachusetts. Cultural Diversity and Ethnic Minority Psychology, 20(1), 16-26.

Sandhu, D. S., \& Asrabadi, H. R. (1994). Development of an acculturative stress scale for international students: Preliminary findings. Psychological Reports, 75, 435448. 
Smith, P. B., Huang, H. J., Harb, C., \& Torres, C. (2012). How distinctive are indigenous ways of achieving influence? A comparative study of guanxi, wasta, jeitinho, and "pulling strings." Journal of Cross-Cultural Psychology, 43(1), 135-150.

Tosta, A. L. A. (2004). The Hispanic and Luso-Brazilian world: Latino, eu? the paradoxical interplay of identity in Brazuca literature. Hispania, 84(3), 576-585.

Yakunina, E. S., Weigold, I. K., Weigold, A., Hercegovac, S., \& Elsayed, N. (2013). International students' personal and multicultural strengths: Reducing acculturative stress and promoting adjustment. Journal of Counseling \& Development, 91(2), 216-223.

Yoon, E., Chang, C. T., Kim, S., Clawson, A., Cleary, S. E., Hansen, M., . . Gomes, A. M. (2013). A meta-analysis of acculturation/enculturation and mental health. Journal of Counseling Psychology, 60(1), 15-30.

SILVIA ALVES NISHIOKA, MA, is a doctoral student in Counseling and Clinical Psychology at Teachers College, Columbia University. Her research interests lie in the area of preventive interventions, adaptation of evidence-based interventions, and multiculturalism. Email: $\underline{\text { san2143@ cc.columbia.edu }}$

DEFNE AKOL, PhD, is a lecturer at Teachers College, Columbia University. Her research interests lie in the area of substance use disorders, eating disorders, the effects of mental and physical illness on families, and professional identity development. Email: da2105@tc.columbia.edu 